W.E. Barrera, J.R. Morales and E.M. Rojas

\title{
A FIXED POINT THEOREM FOR CONTRACTIVE MAPPINGS WITH NONLINEAR COMBINATIONS OF RATIONAL EXPRESSIONS IN $b$-METRIC SPACES
}

\begin{abstract}
In this paper we discuss the existence and uniqueness of fixed points for mappings satisfying several (nonlinear-combinations) contractive inequalities of rational type controlled by altering distance functions. Our results extend several fixed point results in the literature.
\end{abstract}

KEY WORDS: fixed point, rational type, generalized weak contraction, $b$-metric spaces.

AMS Mathematics Subject Classification: 47H10, 54H25, 54D99.

\section{Introduction and preliminaries}

Extensions and generalizations of the Banach contraction principle is a hot research topic in the metric fixed point theory, mainly due to its applicability in diverse areas of knowledge. The seventies of the past century was particular fruitfully in this line of investigation, in particular, Das-Gupta [9] and Jaggi [12] (see also [13]) extended this principle by analyzing the existence of fixed points, on a complete metric space $(M, \rho)$, for mappings satisfying an inequality contraction of the form:

$$
\rho(T x, T y) \leq \Theta(x, y), \quad \text { for all } x, y \in M
$$

where, $\Theta(x, y)$ is a linear combination of elements on the set

$$
\begin{aligned}
\{\rho(x, y), & \frac{\rho(x, T x) \rho(y, T y)}{\rho(x, y)}, \frac{\rho(x, T x) \rho(y, T y)}{\rho(T x, T y)} \\
& \left.\frac{\rho(y, T y)[1+\rho(x, T x)]}{1+\rho(x, y)}, \frac{\rho(x, T x)[1+\rho(y, T y)]}{1+\rho(T x, T y)}\right\} .
\end{aligned}
$$

In recent times, several authors have been generalized the results of Das-Gupta and Jaggi by considering, for instance, partially ordered metric 
spaces, weak contractions of rational type, orbitally metric spaces, triangular $\alpha$-admissible mappings, $(\phi, \psi)$-rational type contractions and common fixed points for pair of rational mappings among others mathematical concepts. See, e.g., $[5,7,11,10,15,16,19]$ and references therein.

The aim of this paper is to prove a fixed point result for mappings satisfying a general contractive inequality of Das-Gupta-Jaggi's type controlled by altering distance functions and some auxiliary functions (see Definition 4 below). This theorem improves and generalizes several fixed points results in the literature.

\subsection{Fixed points in $b$-metric spaces: a motivation}

In 1905, M. Frechet introduced the notion of usual metric space, and due to its importance and applications this concept has been generalized in many different ways, such generalizations include: symmetric spaces, 2-metric spaces, semimetric spaces, G-metric spaces, partial metric spaces, dislocated metric spaces, rectangular metric spaces, b-metric spaces among others. However, as is pointed out in [3], not all these generalizations always produce results which are really new.

Definition 1. Let $M$ be a nonempty set and $s \geq 1$ be a given real number. A function

$$
\rho: M \times M \rightarrow \mathbb{R}_{+}=[0,+\infty),
$$

is called a b-metric provided that for all $x, y, z \in M$,

(a) $\rho(x, y)=0$ iff $x=y$.

(b) $\rho(x, y)=\rho(y, x)$.

(c) $\rho(x, z) \leq s[\rho(x, y)+\rho(y, z)]$.

The pair $(M, \rho)$ is called a b-metric space (also known as a quasimetric space). The number $s \geq 1$ is called a coefficient of $(M, \rho)$.

The class of $b$-metric spaces is larger than that the usual metric spaces, since a $b$-metric space is a usual metric space when $s=1$.

Definition 2. Let $(M, \rho)$ be a b-metric space and let $U$ be a subset of $M$. We say that $U$ is an open subset of $M$ if for each $x \in U$ there exists $r>0$ such that $B(x, r) \subset U$, where

$$
B(x, r)=\{y \in M \mid \rho(x, y)<r\} .
$$

The family of all open subsets of $M$ also is a topology on $M$, see [14, Proposition 1]. Even more, any $b$-metric space $(M, \rho)$ may be canonically viewed as a uniform space whose uniformity has a countable fundamental system of entourages, say, $\left\{(x, y) \in M \times M: \rho(x, y)<n^{-1}\right\}, n \in \mathbb{N}$. 
Notice that as a consequence of the Alexandroff-Urysohn Theorem, the topology induced by a given $b$-metric on a $b$-metric space is metrizable. Quantitative aspects of this metrization are given in the following result due to Macías and Segovia [17].

Theorem 1 (Macías-Segovia). Let $(M, \rho)$ be a b-metric space. Then there exists another b-metric $\rho_{*}$ on $M$ that is equivalent to $\rho$ (in the sense that each is dominated by a fixed multiple of the other) and satisfies the following additional property. If

$$
\alpha=\frac{1}{\log _{2}(s(2 s+1))} \in(0,1)
$$

then, the function $\left(\rho_{*}\right)^{\alpha}: M \times M \longrightarrow[0,+\infty)$ is a metric on $M$ that induces the same topology on $M$ as the original b-metric. In particular, this topology is metrizable.

This theorem plays a prominent role in harmonic analysis, in particular in the setting of spaces of homogeneous type, since the natural framework for analysis in this context is that of $b$-metric spaces.

In functional analysis there are many function spaces of basic importance in partial differential equations that are not Banach but merely quasi-Banach. We recall that a $b$-norm (quasi-norm) $\|\cdot\|$ is a nonnegative function defined on a vector space $M$ that satisfies for each $x, y \in M$ and each $\lambda \in \mathbb{R}$

$$
\|x\|=0 \Leftrightarrow x=0, \quad\|\lambda x\|=|\lambda|\|x\|, \quad\|x+y\| \leq s(\|x\|+\|y\|), \quad s \geq 1 .
$$

The pair $(M,\|\cdot\|)$ is called a quasi-normed space.

Examples of $b$-normed spaces are significant portions of the following familiar scales of spaces: Lebesgue spaces, weak Lebesgue spaces, Lorentz spaces, Hardy spaces, weak Hardy spaces, Lorentz-based Hardy spaces, Besov spaces, Triebel Lizorkin spaces, and weighted versions of these spaces (among many others).

In the context of b-normed spaces, the analogous to the Macías-Segovia Theorem is the Aoki-Rolewicz theorem ([4, 22]) which reads as follows.

Theorem 2 (Aoki-Rolewicz). Let $(M,\|\cdot\|)$ be a b-normed space, then there exists a b-norm $\|\cdot\|_{*}$ on $M$ equivalent to $\|\cdot\|$ which is a p-norm for some $p \in(0,1]$, i.e., it satisfies

$$
\|x+y\|_{*}^{p} \leq\|x\|_{*}^{p}+\|y\|_{*}^{p}, \text { for all } x, y \in M \text {. }
$$

In particular, the topology induced by $\|\cdot\|$ on $M$ is metrizable since it coincides with the topology induced by the distance $d(x, y)=\|x-y\|_{*}^{p}$ for all $x, y \in M$ on $M$. 
For more information on the subject we recommend [18] and references therein.

In the metric fixed point theory, the fact that any $b$-metric space is metrizable gives as a consequence that much of the results given in metric spaces are directly valid in $b$-metric spaces. Thus, in applications, when we are dealing with particular spaces whose metric is difficult to compute, sometimes it is more convenient to verify any contractive inequality by using a $b$-metric instead of the metric on the space as is shown in next example.

\section{Example 1. Let}

$$
\begin{array}{r}
L^{p(\cdot)}(\Omega):=\{f: f: \Omega \longrightarrow \mathbb{R} \text { Lebesgue measurable satisfying } \\
\int_{\Omega}\left|\frac{f(t)}{\lambda}\right|^{p(t)} d t<\infty, \text { for some } \lambda>0, \\
\text { with } p: \Omega \longrightarrow[1,+\infty] \text { measurable }\}
\end{array}
$$

be the so-called variable exponent Lebesgue space. It becomes a Banach space, for $1<p_{-}:=\operatorname{ess~inf}_{\Omega} p(t) \leq p(t) \leq p_{+}:=\operatorname{ess}_{\sup _{\Omega}} f(t)<+\infty$, when it is equipped with the Kolmogorov-Minkowski norm (see, [8, Theorem 2.71])

$$
\|f\|=\inf \left\{\lambda>0: \int_{\Omega}\left|\frac{f(t)}{\lambda}\right|^{p(t)} d t \leq 1\right\}
$$

and it is a $b$-normed space with constant $s=2^{p_{+}}$, with the $b$-norm

$$
\rho(f)=\int_{\Omega}|f(t)|^{p(t)} d t
$$

(See, e.g., [8, Proposition 2.7 and Remark 2.8]). Let us consider the mapping $T: L^{p(\cdot)}(\Omega) \longrightarrow L^{p(\cdot)}(\Omega)$ given by

$$
T f(t)=\frac{f(t)}{2^{1 / p(t)}} .
$$

For any $f, g \in L^{p(\cdot)}(\Omega)$ we have

$$
\begin{aligned}
\rho(T f-T g) & =\int_{\Omega}\left|\frac{f(t)-g(t)}{2^{1 / p(t)}}\right|^{p(t)} d t \\
& =\frac{1}{2} \int_{\Omega}|f(t)-g(t)|^{p(t)} d t=\frac{1}{2} \rho(f-g) .
\end{aligned}
$$


That it, $T$ is a Banach contraction mapping in the b-metric space $\left(L^{p(\cdot)}(\Omega), \rho\right)$. On the other hand,

$$
\begin{aligned}
\|T f-T g\| & =\inf \left\{\lambda>0: \int_{\Omega}\left|\frac{f(t)-g(t)}{\lambda 2^{1 / p(t)}}\right|^{p(t)} d t \leq 1\right\} \\
& =\inf \left\{\lambda>0: \frac{1}{2} \int_{\Omega}\left|\frac{f(t)-g(t)}{\lambda}\right|^{p(t)} d t \leq 1\right\}
\end{aligned}
$$

and

$$
\begin{aligned}
\frac{1}{2}\|f-g\| & =\inf \left\{\frac{1}{2} \lambda>0: \int_{\Omega}\left|\frac{f(t)-g(t)}{\lambda}\right|^{p(t)} d t \leq 1\right\} \\
& =\inf \left\{\mu>0: \int_{\Omega}\left|\frac{f(t)-g(t)}{2 \mu}\right|^{p(t)} d t \leq 1\right\}
\end{aligned}
$$

To prove the equality $\|T f-T g\|=\frac{1}{2}\|f-g\|$, let

$$
\begin{aligned}
& A=\left\{\lambda>0: \frac{1}{2} \int_{\Omega}\left|\frac{f(t)-g(t)}{\lambda}\right|^{p(t)} d t \leq 1\right\}, \\
& B=\left\{\mu>0: \int_{\Omega}\left|\frac{f(t)-g(t)}{2 \mu}\right|^{p(t)} d t \leq 1\right\} .
\end{aligned}
$$

Notice that from the fact $p_{-}>1$, the following inequalities hold:

$$
\begin{aligned}
\int_{\Omega}\left|\frac{f(t)-g(t)}{2 \lambda}\right|^{p(t)} d t & =\int_{\Omega}\left(\frac{1}{2}\right)^{p(t)}\left|\frac{f(t)-g(t)}{\lambda}\right|^{p(t)} d t \\
& \leq \frac{1}{2} \int_{\Omega}\left|\frac{f(t)-g(t)}{\lambda}\right|^{p(t)} d t
\end{aligned}
$$

and

$$
\frac{1}{2} \int_{\Omega}\left|\frac{f(t)-g(t)}{2 \lambda}\right|^{p(t)} d t \leq \int_{\Omega}\left|\frac{f(t)-g(t)}{2 \lambda}\right|^{p(t)} d t .
$$

First, we prove that $A \subset B$. Let $\lambda_{0} \in A$, that is

$$
\frac{1}{2} \int_{\Omega}\left|\frac{f(t)-g(t)}{\lambda_{0}}\right|^{p(t)} d t \leq 1
$$

from (1) we conclude that

$$
\int_{\Omega}\left|\frac{f(t)-g(t)}{2 \lambda_{0}}\right|^{p(t)} d t \leq 1
$$


I.e., $\lambda_{0} \in B$, thus $A \subset B$. Taking infimum we get the inequality $\|T f-T g\| \geq$ $\frac{1}{2}\|f-g\|$. Now we prove $B \subset A$. Let $\mu_{0}:=\frac{\lambda_{0}}{2} \in B$, that means,

$$
\int_{\Omega}\left|\frac{f(t)-g(t)}{2 \mu_{0}}\right|^{p(t)} d t \leq 1
$$

From (2), we have

$$
1 \geq \frac{1}{2} \int_{\Omega}\left|\frac{f(t)-g(t)}{2 \mu_{0}}\right|^{p(t)} d t=\frac{1}{2} \int_{\Omega}\left|\frac{f(t)-g(t)}{\lambda_{0}}\right|^{p(t)} d t .
$$

Therefore, $\mu_{0} \in B$, so $B \subset A$ and $\|T f-T g\| \leq \frac{1}{2}\|f-g\|$. We obtain in this way the equality $\|T f-T g\|=\frac{1}{2}\|f-g\|$.

This example shows that it seems more convenient to consider $L^{p(\cdot)}(\Omega)$ as a $b$-metric space with the $b$-norm $\rho$, than as a metric space with the Kolmogorov-Minkowski norm $\|\cdot\|$, since the computations using the norm are more involve. The reader can verify that the complexity of the computations cannot be easily overcome by considering $L^{p(\cdot)}(\Omega)$ with other well-known norms as, for instance, the associate norm $[8,20]$.

Definition 3. Let $(M, \rho)$ be a b-metric space. A sequence $\left(x_{n}\right)_{n}$ in $M$ is called:

(a) b-convergent if there exists $x \in M$ such that $\lim _{n \rightarrow \infty} \rho\left(x_{n}, x\right)=0$. In this case we write $\lim _{n \rightarrow \infty} x_{n}=x$.

(b) b-Cauchy sequence if

$$
\lim _{n, m \rightarrow \infty} \rho\left(x_{n}, x_{m}\right)=0 .
$$

If every b-Cauchy sequence in $M$ is b-convergent, then $(M, \rho)$ is said to be a complete b-metric space.

From the convergence on a $b$-metric space $(M, \rho)$, the sequential topology in $M$ can be defined as usual. These two topologies coincides [2, Proposition $3.3]$ and as a consequence of this we have for $(M, \rho)$ and $\left(N, \rho^{\prime}\right)$ be two b-metric spaces a function $f: M \rightarrow N$ is $b$-continuous at a point $x \in M$ if only if it is $b$-sequentially continuous at $x$, that is, whenever $\left(x_{n}\right)_{n}$ is $b$-convergent to $x,\left(f\left(x_{n}\right)\right)_{n}$ is $b$-convergent to $f(x)$.

Proposition $1([2])$. Let $(M, \rho)$ be a b-metric space. The following assertions hold:

(a) A b-convergent sequence has a unique limit.

(b) The subsequences of a b-convergent sequence are also $b$-convergent and they are b-convergent to the limit of the original sequence.

(c) Every sequence which is b-convergent is also a b-Cauchy sequence. 
(d) In general a b-metric $\rho: M \times M \rightarrow \mathbb{R}_{+}$with coefficient $s>1$ is not jointly continuous in all its variables and if $\rho$ is continuous in one variable, then $M$ is regular.

The following lemma will be useful in the proof of our main results.

Lemma 1 ([21]). Let $(M, \rho)$ be a b-metric space with coefficient $s \geq 1$. Let $\left(x_{n}\right)_{n}$ be a sequence in $M$ such that $\lim _{n \rightarrow \infty} \rho\left(x_{n}, x_{n+1}\right)=0$. If $\left(x_{n}\right)$ is not a b-Cauchy sequence, then there is $\epsilon>0$ and sequences of positive integers $(n(k))_{k}$ and $(m(k))_{k}$ with

$$
n(k)>m(k)>k
$$

such that $\rho\left(x_{m(k)}, x_{n(k)}\right) \geq \epsilon, \rho\left(x_{m(k)}, x_{n(k)-1}\right)<\epsilon$, and

(a) $\epsilon \leq \limsup _{k \rightarrow \infty} \rho\left(x_{m(k)}, x_{n(k)}\right) \leq \epsilon s$,

(b) $\frac{\epsilon}{s} \leq \limsup _{k \rightarrow \infty} \rho\left(x_{m(k)}, x_{n(k)+1}\right) \leq s^{2} \epsilon$,

(c) $\frac{\epsilon}{s} \leq \limsup _{k \rightarrow \infty} \rho\left(x_{m(k)+1}, x_{n(k)}\right) \leq s^{2} \epsilon$,

(d) $\frac{\epsilon}{s^{2}} \leq \limsup _{k \rightarrow \infty} \rho\left(x_{m(k)+1}, x_{n(k)+1}\right) \leq s^{3} \epsilon$.

\section{On the class of contraction mappings}

In this paper we are going to work with the following classes of functions which have been used in the literature to establish several fixed point results.

$$
\begin{aligned}
\Psi:= & \left\{\varphi: \varphi: \mathbb{R}_{+} \longrightarrow \mathbb{R}_{+}\right. \text {continuous, monotocally non decreasing and } \\
& \text { satisfying } \varphi(0)=0 \Leftrightarrow t=0\} \text { (altering distance functions), } \\
\Phi:= & \left\{(\alpha, \beta, \gamma): \alpha, \beta, \gamma: \mathbb{R}_{+} \longrightarrow[0,1)\right. \text { satisfying } \\
& \left.\limsup _{s \rightarrow t}(\alpha(s))+\limsup _{s \rightarrow t}(\beta(s))+\limsup _{s \rightarrow t}(\gamma(s))<1, \text { for all } t>0\right\} .
\end{aligned}
$$

For a $b$-metric space $(M, \rho)$ and a mapping $T: M \longrightarrow M$, let us introduce the following sets of non-negative coefficients: $\mathcal{M}:=\left\{M_{1}(x, y), M_{2}(x, y)\right.$, $\left.M_{3}(x, y)\right\}$, where

$$
\begin{aligned}
& M_{1}(x, y)=\max \left\{\rho(x, y), \frac{\rho(x, T x) \rho(y, T y)}{\rho(x, y)}\right\}, x \neq y, \\
& M_{2}(x, y)=\max \left\{\rho(x, y), \frac{\rho(y, T y)[1+\rho(x, T x)]}{1+\rho(x, y)}\right\}, \\
& M_{3}(x, y)=\max \left\{\rho(x, y), \frac{\rho(x, T x) \rho(y, T y)}{\rho(x, y)}, \frac{\rho(x, T x) \rho(y, T y)}{\rho(T x, T y)}\right\}, \\
& x \neq y, T x \neq T y .
\end{aligned}
$$


and $\mathcal{N}:=\left\{N_{1}(x, y), N_{2}(x, y), N_{3}(x, y)\right\}$, where

$$
\begin{aligned}
& N_{1}(x, y)=\max \left\{\rho(x, y), \frac{\rho(x, T x) \rho(y, T y)}{\rho(T x, T y)}\right\}, T x \neq T y, \\
& N_{2}(x, y)=\max \left\{\rho(x, y), \frac{\rho(x, T x)[1+\rho(y, T y)]}{1+\rho(T x, T y)}\right\}, \\
& N_{3}(x, y)=\max \left\{\rho(x, y), \frac{\rho(y, T y)[1+\rho(x, T x)]}{1+\rho(x, y)}, \frac{\rho(x, T x)[1+\rho(y, T y)}{1+\rho(T x, T y)}\right\} .
\end{aligned}
$$

By using the sets of functions and $b$-distances defined above, we introduce the following class of contraction mappings.

Definition 4. Let $(M, \rho)$ be a b-metric space with coefficient $s \geq 1$. A mapping $T: M \rightarrow M$ is said to be a $\Psi \times \Phi \times \mathcal{M} \times \mathcal{N}$-contraction if for all $x, y \in M$ it satisfies the following inequality

$$
\begin{aligned}
\psi[s \rho(T x, T y)] \leq & \alpha[\rho(x, y)] \psi[\rho(x, y)]+\beta[\rho(x, y)] \psi[M(x, y)] \\
& +\gamma[\rho(x, y)] \psi[N(x, y)]
\end{aligned}
$$

where $(\psi,(\alpha, \beta, \gamma), M, N) \in \Psi \times \Phi \times \mathcal{M} \times \mathcal{N}$.

Proposition 2. Let $(M, \rho)$ be a b-metric space with coefficient $s \geq 1$ and let $T: M \rightarrow M$ be a mapping. If $T$ is a $\Psi \times \Phi \times \mathcal{M} \times \mathcal{N}$-contraction, then for any $x_{0} \in M$ the sequence $\left(x_{n}\right)_{n}$ defined by $x_{n+1}=T x_{n}, n=0,1,2, \ldots$ satisfies:

(a) $\lim _{n \rightarrow \infty} \rho\left(x_{n}, x_{n+1}\right)=0$,

(b) $\left(x_{n}\right)_{n} \subset M$ is a b-Cauchy sequence.

Proof. To proof $(a)$. Let $x_{0} \in M$ be an arbitrary point and we defined the sequence $\left(x_{n}\right)_{n}$ as follows, $x_{n+1}=T x_{n}, n=0,1,2, \ldots$ Now, using (3) we obtain that

$$
\begin{aligned}
\psi\left[s \rho\left(x_{n}, x_{n+1}\right)\right]= & \psi\left[s \rho\left(T x_{n-1}, T x_{n}\right)\right] \\
\leq & \alpha\left[\rho\left(x_{n-1}, x_{n}\right)\right] \psi\left[\rho\left(x_{n-1}, x_{n}\right)\right] \\
& +\beta\left[\rho\left(x_{n-1}, x_{n}\right)\right] \psi\left[M\left(x_{n-1}, x_{n}\right)\right] \\
& +\gamma\left[\rho\left(x_{n-1}, x_{n}\right)\right] \psi\left[N\left(x_{n-1}, x_{n}\right)\right] .
\end{aligned}
$$

Notice that for $M \in \mathcal{M}$ we have

$$
\begin{aligned}
M_{1}\left(x_{n-1}, x_{n}\right) & =\max \left\{\rho\left(x_{n-1}, x_{n}\right), \frac{\rho\left(x_{n-1}, T x_{n-1}\right) \rho\left(x_{n}, T x_{n}\right)}{\rho\left(x_{n-1}, x_{n}\right)}\right\} \\
& =\max \left\{\rho\left(x_{n-1}, x_{n}\right), \rho\left(x_{n}, x_{n+1}\right)\right\}
\end{aligned}
$$




$$
\begin{aligned}
M_{2}\left(x_{n-1}, x_{n}\right)= & \max \left\{\rho\left(x_{n-1}, x_{n}\right), \frac{\rho\left(x_{n}, T x_{n}\right)\left[1+\rho\left(x_{n-1}, T x_{n-1}\right)\right]}{1+\rho\left(x_{n-1}, x_{n}\right)}\right\} \\
= & \max \left\{\rho\left(x_{n-1}, x_{n}\right), \frac{\rho\left(x_{n}, x_{n+1}\right)\left[1+\rho\left(x_{n-1}, x_{n}\right)\right]}{1+\rho\left(x_{n-1}, x_{n}\right)}\right\} \\
= & \max \left\{\rho\left(x_{n-1}, x_{n}\right), \rho\left(x_{n}, x_{n+1}\right)\right\}, \\
M_{3}\left(x_{n-1}, x_{n}\right)=\max \left\{\rho\left(x_{n-1}, x_{n}\right), \frac{\rho\left(x_{n-1}, T x_{n-1}\right) \rho\left(x_{n}, T x_{n}\right)}{\rho\left(x_{n-1}, x_{n}\right)},\right. & \left.\frac{\rho\left(x_{n-1}, T x_{n-1}\right) \rho\left(x_{n}, T x_{n}\right)}{\rho\left(T x_{n-1}, T x_{n}\right)}\right\} \\
=\max \left\{\rho\left(x_{n-1}, x_{n}\right), \frac{\rho\left(x_{n-1}, x_{n}\right) \rho\left(x_{n}, x_{n+1}\right)}{\rho\left(x_{n-1}, x_{n}\right)},\right. & \left.\frac{\rho\left(x_{n-1}, x_{n}\right) \rho\left(x_{n}, x_{n+1}\right)}{\rho\left(x_{n}, x_{n+1}\right)}\right\} \\
= & \max \left\{\rho\left(x_{n-1}, x_{n}\right), \rho\left(x_{n}, x_{n+1}\right), \rho\left(x_{n-1}, x_{n}\right)\right\} \\
= & \max \left\{\rho\left(x_{n-1}, x_{n}\right), \rho\left(x_{n}, x_{n+1}\right)\right\}
\end{aligned}
$$

and for each for $N \in \mathcal{N}$ we get,

$$
\begin{aligned}
N_{1}\left(x_{n-1}, x_{n}\right)=\max \left\{\rho\left(x_{n-1}, x_{n}\right), \frac{\rho\left(x_{n-1}, x_{n}\right) \rho\left(x_{n}, x_{n+1}\right)}{\rho\left(T x_{n-1}, T x_{n}\right)}\right\} \\
=\max \left\{\rho\left(x_{n-1}, x_{n}\right), \rho\left(x_{n-1}, x_{n}\right)\right\}=\rho\left(x_{n-1}, x_{n}\right), \\
N_{2}\left(x_{n-1}, x_{n}\right)=\max \left\{\rho\left(x_{n-1}, x_{n}\right), \frac{\rho\left(x_{n-1}, T x_{n-1}\right)\left[1+\rho\left(x_{n}, T x_{n}\right)\right]}{1+\rho\left(T x_{n-1}, T x_{n}\right)}\right\} \\
=\max \left\{\rho\left(x_{n-1}, x_{n}\right), \frac{\rho\left(x_{n-1}, x_{n}\right)\left[1+\rho\left(x_{n}, x_{n+1}\right)\right]}{1+\rho\left(x_{n}, x_{n+1}\right)}\right\} \\
=\max \left\{\rho\left(x_{n-1}, x_{n}\right), \rho\left(x_{n-1}, x_{n}\right)\right\}=\rho\left(x_{n-1}, x_{n}\right), \\
N_{3}\left(x_{n-1}, x_{n}\right)=\max \left\{\rho\left(x_{n-1}, x_{n}\right), \frac{\rho\left(x_{n}, T x_{n}\right)\left[1+\rho\left(x_{n-1}, T x_{n-1}\right)\right]}{1+\rho\left(x_{n-1}, x_{n}\right)},\right. \\
\left.\quad \frac{\rho\left(x_{n-1}, T x_{n-1}\right)\left[1+\rho\left(x_{n}, T x_{n}\right)\right.}{1+\rho\left(T x_{n-1}, T x_{n}\right)}\right\} \\
=\max \left\{\rho\left(x_{n-1}, x_{n}\right), \frac{\rho\left(x_{n}, x_{n+1}\right)\left[1+\rho\left(x_{n-1}, x_{n}\right)\right]}{1+\rho\left(x_{n-1}, x_{n}\right)},\right. \\
\left.\quad \frac{\rho\left(x_{n-1}, x_{n}\right)\left[1+\rho\left(x_{n}, x_{n+1}\right)\right.}{1+\rho\left(x_{n}, x_{n+1}\right)}\right\} \\
=\max \left\{\rho\left(x_{n-1}, x_{n}\right), \rho\left(x_{n}, x_{n+1}\right), \rho\left(x_{n-1}, x_{n}\right)\right\} \\
=\max \left\{\rho\left(x_{n-1}, x_{n}\right), \rho\left(x_{n}, x_{n+1}\right)\right\} .
\end{aligned}
$$

From here we have that $N_{1}\left(x_{n-1}, x_{n}\right)=N_{2}\left(x_{n-1}, x_{n}\right)=\rho\left(x_{n-1}, x_{n}\right)$ and the following four situations are possible: 
(a) $N_{3}\left(x_{n-1}, x_{n}\right)=M_{i}\left(x_{n-1}, x_{n}\right)=\rho\left(x_{n-1}, x_{n}\right)$ for all $i \in\{1,2,3\}$,

(b) $N_{3}\left(x_{n-1}, x_{n}\right)=M_{i}\left(x_{n-1}, x_{n}\right)=\rho\left(x_{n}, x_{n+1}\right)$ for all $i \in\{1,2,3\}$,

(c) $N_{3}\left(x_{n-1}, x_{n}\right)=\rho\left(x_{n-1}, x_{n}\right)$ and $M_{i}\left(x_{n-1}, x_{n}\right)=\rho\left(x_{n}, x_{n+1}\right)$ for some (possible all) $i \in\{1,2,3\}$,

(d) $N_{3}\left(x_{n-1}, x_{n}\right)=\rho\left(x_{n}, x_{n+1}\right)$ and $M_{i}\left(x_{n-1}, x_{n}\right)=\rho\left(x_{n-1}, x_{n}\right)$ for some (possible all) $i \in\{1,2,3\}$.

Claim: For each one of the four possible situations above we have that

$$
\psi\left[\rho\left(x_{n}, x_{n+1}\right)\right] \leq c_{n} \psi\left[\rho\left(x_{n-1}, x_{n}\right)\right]
$$

for some $c_{n}$ with $\limsup \sup _{n \rightarrow \infty} c_{n}<1$. To prove this we are going to use that $\alpha(t)+\beta(t)+\gamma(t)<1$ for all $t>0$ and inequality (3). First notice that cases $(a)$ and $(c)$ hold if we replace $N_{3}\left(x_{n-1}, x_{n}\right)$ for $N_{1}\left(x_{n-1}, x_{n}\right)$ and $N_{2}\left(x_{n-1}, x_{n}\right)$ (which complete all the possible situations). Now, we prove the claim for the case $(a)$ :

$$
\begin{aligned}
\psi\left[\rho\left(x_{n}, x_{n+1}\right)\right] \leq & \psi\left[s \rho\left(x_{n}, x_{n+1}\right)\right]=\psi\left[s \rho\left(T x_{n-1}, T x_{n}\right)\right] \\
\leq & \alpha\left[\rho\left(x_{n-1}, x_{n}\right)\right] \psi\left[\rho\left(x_{n-1}, x_{n}\right)\right] \\
& +\beta\left[\rho\left(x_{n-1}, x_{n}\right)\right] \psi\left[\rho\left(x_{n-1}, x_{n}\right)\right] \\
& +\gamma\left[\left(x_{n-1}, x_{n}\right)\right] \psi\left[\rho\left(x_{n-1}, x_{n}\right)\right] \\
= & c_{n}^{1} \psi\left[\rho\left(x_{n-1}, x_{n}\right)\right]
\end{aligned}
$$

where $c_{n}^{1}=\alpha\left[\rho\left(x_{n-1}, x_{n}\right)\right]+\beta\left[\rho\left(x_{n-1}, x_{n}\right)\right]+\gamma\left[\left(x_{n-1}, x_{n}\right)\right]$.

Case (b):

$$
\begin{aligned}
\psi\left[\rho\left(x_{n}, x_{n+1}\right)\right] \leq & \psi\left[s \rho\left(x_{n}, x_{n+1}\right)\right]=\psi\left[s \rho\left(T x_{n-1}, T x_{n}\right)\right] \\
\leq & \alpha\left[\rho\left(x_{n-1}, x_{n}\right)\right] \psi\left[\rho\left(x_{n-1}, x_{n}\right)\right] \\
& +\beta\left[\rho\left(x_{n-1}, x_{n}\right)\right] \psi\left[\rho\left(x_{n}, x_{n+1}\right)\right] \\
& +\gamma\left[\left(x_{n-1}, x_{n}\right)\right] \psi\left[\rho\left(x_{n}, x_{n+1}\right)\right] \\
= & \alpha\left[\rho\left(x_{n-1}, x_{n}\right)\right] \psi\left[\rho\left(x_{n-1}, x_{n}\right)\right]+\left(\beta\left[\rho\left(x_{n-1}, x_{n}\right)\right]\right. \\
& \left.+\gamma\left[\left(x_{n-1}, x_{n}\right)\right]\right) \psi\left[\rho\left(x_{n}, x_{n+1}\right)\right] \\
\leq & \frac{\alpha\left[\rho\left(x_{n-1}, x_{n}\right)\right]}{1-\left(\beta\left[\rho\left(x_{n-1}, x_{n}\right)\right]+\gamma\left[\left(x_{n-1}, x_{n}\right)\right]\right)} \psi\left[\rho\left(x_{n-1}, x_{n}\right)\right] \\
= & c_{n}^{2} \psi\left[\rho\left(x_{n-1}, x_{n}\right)\right] .
\end{aligned}
$$

Here, $c_{n}^{2}=\frac{\alpha\left[\rho\left(x_{n-1}, x_{n}\right)\right]}{1-\left(\beta\left[\rho\left(x_{n-1}, x_{n}\right)\right]+\gamma\left[\left(x_{n-1}, x_{n}\right)\right]\right)}$. 
Case $(c)$ :

$$
\begin{aligned}
\psi\left[\rho\left(x_{n}, x_{n+1}\right)\right] \leq & \psi\left[s \rho\left(x_{n}, x_{n+1}\right)\right]=\psi\left[s \rho\left(T x_{n-1}, T x_{n}\right)\right] \\
\leq & \alpha\left[\rho\left(x_{n-1}, x_{n}\right)\right] \psi\left[\rho\left(x_{n-1}, x_{n}\right)\right] \\
& +\beta\left[\rho\left(x_{n-1}, x_{n}\right)\right] \psi\left[\rho\left(x_{n}, x_{n+1}\right)\right] \\
& +\gamma\left[\left(x_{n-1}, x_{n}\right)\right] \psi\left[\rho\left(x_{n-1}, x_{n}\right)\right] \\
\leq & \frac{\alpha\left[\rho\left(x_{n-1}, x_{n}\right)\right]+\gamma\left[\left(x_{n-1}, x_{n}\right)\right]}{1-\beta\left[\rho\left(x_{n-1}, x_{n}\right)\right]} \psi\left[\rho\left(x_{n-1}, x_{n}\right)\right] \\
= & c_{n}^{3} \psi\left[\rho\left(x_{n-1}, x_{n}\right)\right],
\end{aligned}
$$

with $c_{n}^{3}=\frac{\alpha\left[\rho\left(x_{n-1}, x_{n}\right)\right]+\gamma\left[\left(x_{n-1}, x_{n}\right)\right]}{1-\beta\left[\rho\left(x_{n-1}, x_{n}\right)\right]}$.

Case $(d)$ : Similar computations as the case $(c)$ allow us to conclude that

$$
\begin{aligned}
\psi\left[\rho\left(x_{n}, x_{n+1}\right)\right] & \leq \frac{\alpha\left[\rho\left(x_{n-1}, x_{n}\right)\right]+\beta\left[\left(x_{n-1}, x_{n}\right)\right]}{1-\gamma\left[\rho\left(x_{n-1}, x_{n}\right)\right]} \psi\left[\rho\left(x_{n-1}, x_{n}\right)\right] \\
& =c_{n}^{4} \psi\left[\rho\left(x_{n-1}, x_{n}\right)\right] .
\end{aligned}
$$

In this case, $c_{n}^{4}=\frac{\alpha\left[\rho\left(x_{n-1}, x_{n}\right)\right]+\beta\left[\left(x_{n-1}, x_{n}\right)\right]}{1-\gamma\left[\rho\left(x_{n-1}, x_{n}\right)\right]}$.

The conclusion is obtained by considering $c_{n}=\max \left\{c_{n}^{i},: i=1,2,3,4\right\}$, taking into account that $(\alpha, \beta, \gamma) \in \Phi$. Now, since $\psi \in \Psi$, we have that $\left(\rho\left(x_{n-1}, x_{n}\right)\right)_{n}$ is a non increasing sequence of positive real numbers, then there exists $a \geq 0$ such that $\lim _{n \rightarrow \infty} \rho\left(x_{n}, x_{n+1}\right)=a$. If we suppose that $a>0$, by letting $n \rightarrow \infty$ in (5) we conclude that $\psi(a)<\psi(a)$ which is not possible, therefore $a=0$ since in this case inequality (4) becomes an equality.

Now we are going to prove $(b)$. We want to show that $\left(x_{n}\right)_{n} \subset M$ is a $b$-Cauchy sequence. According to Lemma 1 , if $\left(x_{n}\right)_{n}$ is not a $b$-Cauchy sequence, then there exist $\epsilon>0$ and two subsequences $\left(x_{m(k)}\right)_{k}$ and $\left(x_{n(k)}\right)_{k}$ of $\left(x_{n}\right)_{n}$ with $n(k)>m(k)>k$ such that $\rho\left(x_{m(k)}, x_{n(k)}\right) \geq \epsilon$ and $\rho\left(x_{m(k)}\right.$, $\left.x_{n(k)-1}\right)<\epsilon$ for all positive numbers $k$.

Putting $x=x_{m(k)}$ and $y=x_{n(k)}$ in $(3)$ for $i, j \in\{1,2,3\}$, we obtain

$$
\begin{aligned}
(6) 0<\psi(s \epsilon) \leq & \limsup _{k \rightarrow \infty} \psi\left[s \rho\left(x_{m(k)}, x_{n(k)}\right)\right] \\
= & \limsup _{k \rightarrow \infty} \psi\left[s \rho\left(T x_{m(k)-1}, T x_{n(k)-1}\right)\right] \\
\leq & \limsup _{k \rightarrow \infty} \alpha\left[\rho\left(x_{m(k)-1}, x_{n(k)-1}\right)\right] \psi\left[\rho\left(x_{m(k)-1}, x_{n(k)-1}\right)\right] \\
& +\limsup _{k \rightarrow \infty} \beta\left[\rho\left(x_{m(k)-1}, x_{n(k)-1}\right)\right] \psi\left[M_{i}\left(x_{m(k)-1}, x_{n(k)-1}\right)\right] \\
& +\limsup _{k \rightarrow \infty} \gamma\left[\rho\left(x_{m(k)-1}, x_{n(k)-1}\right)\right] \psi\left[N_{j}\left(x_{m(k)-1}, x_{n(k)-1}\right)\right],
\end{aligned}
$$


where for each $M_{i} \in \mathcal{M}, i \in\{1,2,3\}$, we have

$$
\begin{aligned}
& M_{1}\left(x_{m(k)-1}, x_{n(k)-1}\right)=\max \left\{\rho\left(x_{m(k)-1}, x_{n(k)-1}\right)\right. \text {, } \\
& \left.\frac{\rho\left(x_{m(k)-1}, T x_{m(k)-1}\right) \rho\left(x_{n(k)-1}, T x_{n(k)-1}\right)}{\rho\left(x_{m(k)-1}, x_{n(k)-1}\right)}\right\} \\
& =\max \left\{\rho\left(x_{m(k)-1}, x_{n(k)-1}\right)\right. \text {, } \\
& \left.\frac{\rho\left(x_{m(k)-1}, x_{m(k)}\right) \rho\left(x_{n(k)-1}, x_{n(k)}\right)}{\rho\left(x_{m(k)-1}, x_{n(k)-1}\right)}\right\}, \\
& M_{2}\left(x_{m(k)-1}, x_{n(k)-1}\right)=\max \left\{\rho\left(x_{m(k)-1}, x_{n(k)-1}\right)\right. \text {, } \\
& \left.\frac{\rho\left(x_{n(k)-1}, T x_{n(k)-1}\right)\left[1+\rho\left(x_{m(k)-1}, T x_{m(k)-1}\right)\right]}{1+\rho\left(x_{m(k)-1}, x_{n(k)-1}\right)}\right\} \\
& =\max \left\{\rho\left(x_{m(k)-1}, x_{n(k)-1}\right)\right. \text {, } \\
& \left.\frac{\rho\left(x_{n(k)-1}, x_{n(k)}\right)\left[1+\rho\left(x_{m(k)-1}, x_{m(k)}\right)\right]}{1+\rho\left(x_{m(k)-1}, x_{n(k)-1}\right)}\right\}, \\
& M_{3}\left(x_{m(k)-1}, x_{n(k)-1}\right)=\max \left\{\rho\left(x_{m(k)-1}, x_{n(k)-1}\right),\right. \\
& \frac{\rho\left(x_{m(k)-1}, T x_{m(k)-1}\right) \rho\left(x_{n(k)-1}, T x_{n(k)-1}\right)}{\rho\left(x_{m(k)-1}, x_{n(k)-1}\right)}, \\
& \left.\frac{\rho\left(x_{m(k)-1}, T x_{m(k)-1}\right) \rho\left(x_{n(k)-1}, T x_{n(k)-1}\right)}{\rho\left(T x_{m(k)-1}, T x_{n(k)-1}\right)}\right\} \\
& =\max \left\{\rho\left(x_{m(k)-1}, x_{n(k)-1}\right)\right. \text {, } \\
& \frac{\rho\left(x_{m(k)-1}, x_{m(k)}\right) \rho\left(x_{n(k)-1}, x_{n(k)}\right)}{\rho\left(x_{m(k)-1}, x_{n(k)-1}\right)}, \\
& \left.\frac{\rho\left(x_{m(k)-1}, x_{m(k)}\right) \rho\left(x_{n(k)-1}, x_{n(k)}\right)}{\rho\left(x_{m(k)}, x_{n(k)}\right)}\right\}
\end{aligned}
$$

and for each $N_{j} \in \mathcal{N}, j \in\{1,2,3\}$

$$
\begin{aligned}
N_{1}\left(x_{m(k)-1}, x_{n(k)-1}\right)=\max \{ & \rho\left(x_{m(k)-1}, x_{n(k)-1}\right), \\
& \left.\frac{\rho\left(x_{m(k)-1}, T x_{m(k)-1}\right) \rho\left(x_{n(k)-1}, T x_{n(k)-1}\right)}{\rho\left(T x_{m(k)-1}, T x_{n(k)-1}\right)}\right\} \\
=\max \left\{\rho\left(x_{m(k)-1}, x_{n(k)-1}\right),\right. & \left.\frac{\rho\left(x_{m(k)-1}, x_{m(k)}\right) \rho\left(x_{n(k)-1}, x_{n(k)}\right)}{\rho\left(x_{m(k)}, x_{n(k)}\right)}\right\}, \\
N_{2}\left(x_{m(k)-1}, x_{n(k)-1}\right)=\max \left\{\rho\left(x_{m(k)-1}, x_{n(k)-1}\right),\right. & \left.\frac{\rho\left(x_{m(k)-1}, T x_{m(k)-1}\right)\left[1+\rho\left(x_{n(k)-1}, T x_{n(k)-1}\right)\right]}{1+\rho\left(x_{m(k)}, x_{n(k)}\right)}\right\}
\end{aligned}
$$




$$
\begin{aligned}
=\max & \left\{\rho\left(x_{m(k)-1}, x_{n(k)-1}\right),\right. \\
& \left.\frac{\rho\left(x_{m(k)-1}, x_{m(k)}\right)\left[1+\rho\left(x_{n(k)-1}, x_{n(k)}\right)\right]}{1+\rho\left(x_{m(k)}, x_{n(k)}\right)}\right\}, \\
N_{3}\left(x_{m(k)-1}, x_{n(k)-1}\right)=\max \left\{\rho\left(x_{m(k)-1}, x_{n(k)-1}\right),\right. & \left.\left.\frac{\rho\left(x_{n(k)-1}, T x_{n(k)-1}\right)\left[1+\rho\left(x_{m(k)-1}, T x_{m(k)-1}\right)\right]}{1+\rho\left(x_{m(k)-1}, x_{n(k)-1}\right)}, T x_{n(k)-1}\right)\right\} \\
=\max \left\{\frac{\rho\left(x_{m(k)-1}, T x_{m(k)-1}\right)\left[1+\rho\left(x_{n(k)-1}, T x_{m(k)-1}, x_{n(k)-1}\right),\right.}{1+\rho\left(T x_{m(k)-1}, T x_{n(k)-1}\right)}\right. & \frac{\rho\left(x_{n(k)-1}, x_{n(k)}\right)\left[1+\rho\left(x_{m(k)-1}, x_{m(k)}\right)\right]}{1+\rho\left(x_{m(k)-1}, x_{n(k)-1}\right)}, \\
& \left.\frac{\rho\left(x_{m(k)-1}, x_{m(k)}\right)\left[1+\rho\left(x_{n(k)-1}, x_{n(k)}\right)\right.}{1+\rho\left(x_{m(k)}, x_{n(k)}\right)}\right\} .
\end{aligned}
$$

Since $\lim _{n \rightarrow \infty} \rho\left(x_{n}, x_{n+1}\right)=0$, by the Lemma 1 we have

$$
\begin{aligned}
& \limsup _{k \rightarrow \infty} M_{i}\left(x_{m(k)-1}, x_{n(k)-1}\right) \leq s \epsilon, \quad i \in\{1,2,3\} \\
& \text { and } \\
& \underset{k \rightarrow \infty}{\limsup } N_{j}\left(x_{m(k)-1}, x_{n(k)-1}\right) \leq s \epsilon, \quad j \in\{1,2,3\} .
\end{aligned}
$$

Using (6) and (7) we get

$$
0<\psi(s \epsilon)<\psi(s \epsilon)
$$

which is a contradiction. Therefore $\left(x_{n}\right)_{n}$ is a $b$-Cauchy sequence in $M$.

\section{A fixed point theorem for $\Psi \times \Phi \times \mathcal{M} \times \mathcal{N}$-contractions}

In this section we present our theorem of existence and uniqueness of fixed points for mappings of the class of $\Psi \times \Phi \times \mathcal{M} \times \mathcal{N}$-contractions.

Theorem 3. Let $(M, \rho)$ be a complete b-metric space with coefficient $s \geq 1$ and $T: M \rightarrow M$ be a b-continuous $\Psi \times \Phi \times \mathcal{M} \times \mathcal{N}$-contraction. Then $T$ has a unique fixed point.

Proof. Let $x_{0} \in M$ be an arbitrary point and we define the sequence $\left(x_{n}\right)_{n}$ as before

$$
x_{n+1}=T x_{n}, \quad n=0,1,2, \ldots
$$


From Proposition 2, we know that $\left(x_{n}\right)_{n}$ is a $b$-Cauchy sequence in $M$ and due to the fact that $M$ is a complete $b$-metric space, there exists a unique $z \in M$ such that

$$
\lim _{n \rightarrow \infty} \rho\left(x_{n}, z\right)=0
$$

Since $T$ is $b$-continuous (i.e., sequentially continuous) then $\lim _{n \rightarrow \infty} \rho\left(T x_{n}, T z\right)$ $=0$. On the other hand, from the triangle inequality, we have

$$
\begin{aligned}
\rho(z, T z) & \leq s \rho\left(z, T x_{n}\right)+s \rho\left(T x_{n}, T z\right) \\
& =s \rho\left(z, x_{n+1}\right)+s \rho\left(T x_{n}, T z\right) .
\end{aligned}
$$

Taking limits as $n \rightarrow \infty$ we conclude that $\rho(z, T z)=0$, thus $T z=z$, i.e., $z$ is a fixed point of $T$.

Now, we want to prove that the fixed point of $T$ is unique. In fact, suppose that there exists $w \in M$ such that $w \neq z$ and $T w=w$. Therefore,

$$
\begin{aligned}
\psi[\rho(z, w)] \leq & \psi[s \rho(z, w)]=\psi[s \rho(T z, T w)] \\
\leq & \alpha[\rho(z, w)] \psi[\rho(z, w)] \\
& +\beta[\rho(z, w)] \psi\left[M_{i}(z, w)\right] \\
& +\gamma[\rho(z, w)] \psi\left[N_{j}(z, w)\right], \text { for } i, j \in\{1,2,3\} .
\end{aligned}
$$

It is not difficult to see that

$$
M_{i}(z, w)=\rho(z, w) \quad \text { and } \quad N_{j}(z, w)=\rho(z, w), \quad i, j \in\{1,2,3\}
$$

Hence,

$$
\psi[\rho(z, w)] \leq(\alpha[\rho(z, w)]+\beta[\rho(z, w)]+\gamma[\rho(z, w)]) \psi[\rho(z, w)]
$$

from here it follows that,

$$
\psi[\rho(z, w)]<\psi[\rho(z, w)]
$$

which is a contradiction, therefore $z=w$.

We can drop the continuity of the mapping $T$ in the following particular case.

Theorem 4. Let $(M, \rho)$ be a complete b-metric space with coefficient $s \geq 1$ and $T: M \rightarrow M$ be $a \Psi \times \Phi \times\left\{M_{2}(x, y)\right\} \times \mathcal{N}$-contraction. Then $T$ has a unique fixed point.

Proof. Let $x_{0} \in M$ be an arbitrary point and, as before, let the sequence $\left(x_{n}\right)_{n}$ given by $x_{n+1}=T x_{n}, n=0,1,2, \ldots$ 
By Proposition 2 we have that $\left(x_{n}\right)_{n}$ is a $b$-Cauchy sequence in $M$ and it satisfies that $\lim _{n \rightarrow \infty} \rho\left(x_{n}, x_{n+1}\right)=0$. Since $M$ is a complete $b$-metric space, there exists $z \in M$ such that $\lim _{n \rightarrow \infty} \rho\left(x_{n}, z\right)=0$.

On the other hand, since $T \in \Psi \times \Phi \times\left\{M_{2}(x, y)\right\} \times \mathcal{N}$, notice that

$$
\begin{aligned}
\lim _{n \rightarrow \infty} \psi\left[s \rho\left(T x_{n}, T z\right)\right] \leq \lim _{n \rightarrow \infty} & \left(\alpha\left[\rho\left(x_{n}, z\right)\right] \psi\left[\rho\left(x_{n}, z\right)\right]\right. \\
& +\beta\left[\rho\left(x_{n}, z\right)\right] \psi\left[M_{2}\left(x_{n}, z\right)\right] \\
& \left.+\gamma\left[\rho\left(x_{n}, z\right)\right] \psi\left[N\left(x_{n}, z\right)\right]\right),
\end{aligned}
$$

where, from Proposition 2, we obtain:

$$
\begin{aligned}
\lim _{n \rightarrow \infty} \psi\left[\rho\left(x_{n}, z\right)\right] & =0, \\
\lim _{n \rightarrow \infty} \psi\left[M_{2}\left(x_{n}, z\right)\right] & =\psi[\rho(z, T z)], \\
\lim _{n \rightarrow \infty} \psi\left[N_{1}\left(x_{n}, z\right)\right] & =0, \\
\lim _{n \rightarrow \infty} \psi\left[N_{2}\left(x_{n}, z\right)\right] & =0, \\
\lim _{n \rightarrow \infty} \psi\left[N_{3}\left(x_{n}, z\right)\right] & =\psi[\rho(z, T z)] .
\end{aligned}
$$

Now, from the triangle inequality and since $\psi \in \Psi$ then,

$$
\begin{aligned}
\psi[\rho(z, T z)]= & \lim _{n \rightarrow \infty} \psi[\rho(z, T z)] \leq \lim _{n \rightarrow \infty}\left(\psi\left[s \rho\left(z, T x_{n}\right)+s \rho\left(T x_{n}, T z\right)\right]\right) \\
= & \psi\left[\lim _{n \rightarrow \infty} s \rho\left(T x_{n}, T z\right)\right]=\lim _{n \rightarrow \infty} \psi\left[s \rho\left(T x_{n}, T z\right)\right] \\
\leq & \lim _{n \rightarrow \infty} \alpha\left[\rho\left(x_{n}, z\right)\right] 0+\lim _{n \rightarrow \infty} \beta\left[\rho\left(x_{n}, z\right)\right] \psi[\rho(z, T z)] \\
& \left.+\lim _{n \rightarrow \infty} \gamma\left[\rho\left(x_{n}, z\right)\right] \lim _{n \rightarrow \infty} \psi\left[N\left(x_{n}, z\right)\right]\right) .
\end{aligned}
$$

If in the inequality above $N\left(x_{n}, z\right)$ is equal to $N_{1}\left(x_{n}, z\right)$ or $N_{2}\left(x_{n}, z\right)$ we obtain

$$
\psi[\rho(z, T z)] \leq \lim _{n \rightarrow \infty} \beta\left[\rho\left(x_{n}, z\right)\right] \psi[\rho(z, T z)]<\psi[\rho(z, T z)]
$$

And in the case $N\left(x_{n}, z\right)=N_{3}\left(x_{n}, z\right)$ we have

$$
\begin{aligned}
\psi[\rho(z, T z)] & \leq\left(\lim _{n \rightarrow \infty} \beta\left[\rho\left(x_{n}, z\right)\right]+\lim _{n \rightarrow \infty} \gamma\left[\rho\left(x_{n}, z\right)\right]\right) \psi[\rho(z, T z)] \\
& <\psi[\rho(z, T z)] .
\end{aligned}
$$

In both cases we get a contradiction, therefore $\psi[\rho(z, T z)]=0$ and so $T z=$ $z$. The uniqueness of the fixed point runs similarly as the proof of the Theorem 3.

In 2002, A. Branciari [6] extended the Banach contraction principle by considering mappings satisfying contractive conditions of integral type. Since 
then, there has been increasing interest in the study of fixed points and common fixed points of this kind of mappings. In next result, as a consequence of Theorem 3, we will prove a fixed point result for a class of mappings of integral type.

Let the set of functions

$$
\begin{aligned}
& \Upsilon:=\left\{\varphi: \varphi: \mathbb{R}_{+} \longrightarrow \mathbb{R}_{+}\right. \text {is Lebesgue integrable on each compact } \\
&\text { set of } \left.\mathbb{R}_{+} \text {and } \int_{0}^{\epsilon} \varphi(t) d t>0 \text { for each } \epsilon>0\right\} .
\end{aligned}
$$

Corollary 1. Let $(M, \rho)$ be a complete b-metric space with coefficient $s \geq 1$ and let $T: M \rightarrow M$ be a continuous function satisfying the following inequality for all $x, y \in M$

$$
\begin{aligned}
\int_{0}^{\psi[s \rho(T x, T y)]} \varphi(t) d t \leq & \alpha[\rho(x, y)] \int_{0}^{\psi[\rho(x, y)]} \varphi(t) d t \\
& +\beta[\rho(x, y)] \int_{0}^{\psi[M(x, y)]} \varphi(t) d t+\gamma[\rho(x, y)] \int_{0}^{\psi[N(x, y)]} \varphi(t) d t
\end{aligned}
$$

where $(\psi, \varphi,(\alpha, \beta, \gamma), M, N) \in \Psi \times \Upsilon \times \Phi \times \mathcal{M} \times \mathcal{N}$. Then $T$ has a unique fixed point.

Proof. Let $\varphi \in \Upsilon$, we define

$$
\psi_{0}(s)=\int_{0}^{s} \varphi(t) d t, \quad s \in \mathbb{R}_{+} .
$$

$\psi_{0} \in \Psi$ (see, e.g., [1]). Using the function $\psi_{0}$, inequality (8) becomes

$$
\begin{aligned}
\psi_{0}[\psi(s \rho(T x, T y))] \leq & \alpha[\rho(x, y)] \psi_{0}[\psi \rho(x, y)] \\
& +\beta[\rho(x, y)] \psi_{0}[\psi(M(x, y))] \\
& +\gamma[\rho(x, y)] \psi_{0}[\psi(N(x, y))] .
\end{aligned}
$$

Since $\psi_{0} \circ \psi \in \Psi$, then, from Theorem 3, T has a unique fixed point.

Acknowledgments. The authors are thankful to the referee for the very constructive comments and suggestions.

\section{References}

[1] Abbas M., Khan M.A., Common fixed point theorem of two mappings satisfying a generalized weak contractive condition, International Journal of Mathematics and Mathematical Sciences, vol. 2009, Article ID 131068, 9 pages, 2009. 
[2] An T.V., Tuyen L.Q., Dung V., Stone-type theorem on b-metric spaces and applications, Topology and its Applications, 185-186(2015), 50-64.

[3] An T.V., Dung N.V., Kadelburg Z., Various generalizations of metric spaces and fixed point theorems, Rev. Real Acad. Ciencias Ex. Fis. y Nat. Serie A., 109(1)(2015), 175-198.

[4] Aoki T., Locally bounded topological spaces, Proc. Jpn. Acad. Tokyo, 18(1942), 588-594.

[5] Arshad M., Karapinar E., Ahmad J., Some unique fixed point theorems for rational contractions in partially ordered metric spaces, Journal of Inequalities and Applications 2013, 248(2013).

[6] BRANCIARI A., A fixed point theorem for mappings satisfying a general contractive condition of integral type, Int. J. Math. Math. Sci., 29(9)(2002), 531-536.

[7] Chandoka S., Choudhuryb B.S., Metiyac N., Fixed point results in ordered metric spaces for rational type expressions with auxiliary functions, Journal of the Egyptian Mathematical Society, 23(1)(2015), 95-101.

[8] Cruz-Uribe D.V., Fiorenza A., Variable Lebesgue spaces, Foundations and harmonic analysis, Appl. Num. Harm. Anal., Birkhäuser, Basel, 2013, 317 pp.

[9] Dass B.K., Gupta S., An extension of Banach contracion principle through rational expression, Indian J. Pure appl. Math., 6(1975), 1455-1458.

[10] Harjani J., López B., Sadarangani K., A fixed point theorem for mappings satisfying a contractive condition of rational type on a partially ordered metric space, Abstract and Applied Analysis, Volume 2010 (2010), Article ID 190701, 8 pages.

[11] Hussain N., Parvaneh V., Samet B., Vetro C., Some fixed point theorems for generalized contractive mappings in complete metric spaces, Fixed Point Theory and Applications 2015, 185(2015).

[12] Jaggi D.S., Some unique fixed point theorems, Indian J. Pure Appl. Math., 8(2)(1977), 223-230.

[13] JAGGi D.S., DAs B.K., An extension of Banach's fixed point theorem through rational expression, Bull. Cal. Math. Soc., 72(1980), 261-264.

[14] Khamsi M.A., Hussain N., KKM mappings in metric type spaces, Nonlinear Anal., 7(9)(2010), 3123-3129.

[15] Luong N.V., Thuan N.X., Fixed point theorem for generalized weak contractions satisfying rational expressions in ordered metric spaces, Fixed Point Theory Appl., 2011, Art. ID 46, (2011).

[16] Nashine H.K., Sintunavarat W., Kadelburg Z., Kumam P., Fixed point theorems in orbitally 0 -complete partial metric spaces via rational contractive conditions, Afrika Matematika, 26(5)(2015), 1121-1136.

[17] Macías R.A., Segovia C., Lipschitz functions on spaces of homogeneous type, Adv. Math., 33(1979), 257-270.

[18] Mitrea D., Mitrea I., Mitrea M., Monniaux S., Groupoid Metrization Theory with Applications to Analysis on Quasi-Metric Spaces and Functional Analysis, Appl. Num. Harm. Anal., Birkhäuser Basel, 2013, 486 pp.

[19] Morales J.R., Rojas E.M., Contractive mappings of rational type controlled by minimal requirements functions, Afr. Mat., 27(2016), 65-77. 
[20] Rafeiro H., Rojas E., Espacios de Lebesgue con Exponente Variable: Un espacio de Banach de funciones medibles (Spanish), IVIC-Instituto Venezolano de Investigaciones Cientificas, 2014, pp. XI+136.

[21] Roshan J.R., Parnaveh V., Kadelburg Z., Common fixed point theorems for weakly Isotone increasing mappings in ordered $b$-metric spaces, $J$. Nonlinear Sci and Appl., 7(2014), 229-245.

[22] Rolewicz S., On a certain class of linear metric spaces, Bull. Acad. Polon. Sci. Sér. Sci. Math. Astronom. Phys., 5(1957), 471-473.

W.E. BARRERA

Departamento de Física y Matemáticas

NURR, UlA, Trujillo, VeneZuela

e-mail: wilmereb@ula.ve

J.R. MORALES

Departamento de Matemáticas

UNIVERSIDAD DE LOS ANDES

MÉRIDA, VENEZUELA

e-mail: moralesj@ula.ve

E.M. Rojas

Departamento de Matemáticas

Universidad NACIONAL DE COLOMBIA

Bogotá, Colombia

e-mail: emrojass@unal.edu.co

Received on 11.11.2016 and, in revised form, on 28.03.201\%. 